\section{Living in three dimensions}

$\mathrm{T}$ he Canadian Thoracic Society (CTS) has always had a dual identity, and lived out its mission and mandate in two dimensions: as the advisory medical section of the Canadian Lung Association; and as the national specialty society for respirology in Canada. These two dimensions have coexisted side by side since 1958, when the CTS was formally established. The maturation of the society as a professional association representing the specialty recognized by the Royal College, coupled with the expansion of the nature and scope of our work and collaborations, has resulted in the CTS incorporating as a not-forprofit corporation. In addition to continuing to operate on these two planes, we are now operating in a third dimension to complete the post-incorporation steps that will result in the CTS being a fullfledged independent organization.

The term 'third dimension' conjures up the science fiction image of an alternative universe or other imagined plane of existence. It can also be defined as something that heightens the reality, vividness or significance of a factual account, or sequence of happenings. I can attest that 'heightened reality' is certainly our day-to-day experience now as we navigate the legal, financial and governance processes to firmly establish the CTS in its new organizational form, align our resources with our strategic priorities and redefine our relationship with the Lung Association federation.

While the leadership of CTS systematically works through the legal and administrative requirements leading to full autonomy, we have not lost sight of the importance of service to members and the wider respiratory community through education and knowledge translation. In parallel with our organizational development processes, staff and leading volunteer members have been diligently moving forward with a number of key initiatives that further CTS' strategic directions. These include:

- Development of a guidelines continuing medical education (CME) 'app' by the Asthma Clinical Assembly in collaboration with McGill University that will allow primary care and specialist physicians to both apply and provide feedback on the CTS' evidence-based recommendations;

- Development of a post-discharge pathway for chronic obstructive pulmonay disease (COPD) patients by the COPD Clinical Assembly in partnership with the Respiratory Health Strategic Clinical Network of Alberta;

- Preparation of three position papers to support diagnosis and treatment of interstitial lung disease (ILD) by the ILD Clinical Assembly;

- Creation of a CME program on ILD, co-developed in collaboration with Boehringer Ingelheim;

- Updating of the chronic thromboembolic pulmonary hypertension (CTEPH) guideline by the Pulmonary Vascular Disease Clinical Assembly;

- Delivery of a series of accredited webinars this fall, featuring CTS member experts in adult and pediatric asthma, pulmonary rehabilitation and sleep medicine;

- Development of an interactive slide deck for CME on the prevention of acute exacerbation of COPD and creation of a CTS Speakers Bureau, which will be launched in January 2016; and

- Finalization of an excellent scientific program for the 2016 edition of the Canadian Respiratory Conference (kudos to CTS Scientific Chair Paul Hernandez and his committee!).

\section{Vivre en trois dimensions}

T a Société canadienne de thoracologie a toujours porté une double Lidentité et réalisé sa mission et son mandat à deux égards - en tant que section médicale consultative de l'Association pulmonaire du Canada et comme société nationale de spécialité en pneumologie. Ces deux dimensions ont évolué côte à côte depuis la fondation de la SCT en 1958. Or la maturation de la société en tant qu'association professionnelle représentant la spécialité et reconnue par le Collège royal, couplée à l'expansion de la nature et de la portée de ses travaux et collaborations, ont récemment conduit à sa constitution en société sans but lucratif. En plus de poursuivre nos activités sur ces deux fronts, nous nous activons à présent dans une troisième dimension pour franchir les étapes post-incorporation qui feront de la SCT un organisme indépendant à part entière.

L'expression « troisième dimension » évoque des images d'univers parallèles ou d'autres plans d'existence dignes de la science-fiction. On la définit également comme un élément qui rehausse la réalité, la vivacité ou la signification d'un récit factuel ou d'une séquence d'événements. Je peux attester du fait que nous rencontrons cette « réalité augmentée " au quotidien, à présent que nous naviguons les processus juridiques, financiers et de gouvernance pour établir plus solidement la SCT dans sa nouvelle forme organisationnelle, alignons nos ressources selon nos priorités stratégiques et redéfinissons notre relation avec la fédération de l'Association pulmonaire.

Toutefois, pendant que la direction de la SCT s'occupe systématiquement des exigences juridiques et administratives d'une autonomie complète, nous ne perdons pas de vue l'importance du service aux membres et à l'ensemble de la communauté respiratoire par l'éducation et l'application des connaissances. Parallèlement à nos processus de développement organisationnel, des employés et des membres bénévoles clés travaillent assidûment à diverses initiatives d'importance pour faire avancer les orientations stratégiques de la SCT. Notamment :

- Le développement par l'Assemblée clinique sur l'asthme d'une « appli » de lignes directrices pour la formation médicale continue, en collaboration avec l'Université McGill, qui permettra aux médecins de soins primaires et spécialistes de fournir des rétroactions sur la mise en œuvre et l'évaluation de recommandations fondées sur des données probantes;

- L'élaboration par l'Assemblée clinique sur la MPOC d'un parcours post-hospitalisation pour les patients en MPOC, en partenariat avec le Réseau clinique stratégique sur la santé respiratoire d l'Alberta;

- La préparation par l'Assemblée clinique sur la maladie pulmonaire interstitielle de trois documents de position appuyant le diagnostic et le traitement de la MPI;

- Le codéveloppement d'un programme de formation médicale continue en maladie pulmonaire interstitielle, en collaboration avec Boehringer Ingelheim;

- La mise à jour des lignes directrices sur l'HPTC par l'Assemblée clinique sur les maladies vasculaires pulmonaires;

- La présentation, cet automne, d'une série de webinaires agréés et offerts par des membres de la SCT experts de l'asthme adulte et pédiatrique, de la réadaptation pulmonaire et de la médecine du sommeil;

- Le développement d'un diaporama interactif pour la formation médicale continue sur la prévention des EAMPOC et la création d'un Bureau des conférenciers de la SCT (qui sera inauguré en janvier 2016); et 
Thanks to CTS members who have given time and expertise in support of all of these important projects and to CTS Manager of Education and Knowledge Translation, Kristen Curren, for her able coordination.

We have also continued with some of our highly valued traditional programs such as the annual CTS Scientific Program at CHEST and the CTS Honorary Lecture. This year, the CTS Executive is pleased to announce that Dr Paul M O'Byrne, Distinguished Professor at McMaster University (Hamilton, Ontario), will present the 2015 CTS Honorary Lecture at CHEST. Previously known as the Christie Memorial Lecture, this award recognizes significant contributions to the development of research, education or clinical excellence in the field of Canadian respiratory medicine and their contribution to the CTS. Congratulations Dr O'Byrne!

While the CTS Board and Executive must continue to be preoccupied with the very necessary 'third dimension' and the future of CTS, it is crucial to continue important traditions such as these that celebrate the contributions of our members to the profession and to the improvement of lung health in Canada. On behalf of the CTS, I express my deep appreciation to our Director, Janet Sutherland, for her tireless dedication to our society, and to members of our standing committees and Clinical Assemblies who are on the frontlines of this excellent work!
- La finalisation d'un excellent programme scientifique pour l'édition 2016 du Congrès canadien sur la santé respiratoire (félicitations au président scientifique de la SCT, Paul Hernandez, ainsi qu'à son comité).

Merci aux membres de la SCT qui ont fait don de leur temps et de leur expertise pour appuyer ces projets importants, et à Kristen Curren, gestionnaire de l'éducation et de l'application du savoir de la SCT, pour son indispensable coordination.

Nous avons également poursuivi certains de nos programmes réguliers très appréciés comme le Programme scientifique annuel de la SCT au Congrès CHEST et la Conférence honorifique de la SCT. Cette année, le Comité exécutif de la SCT est heureux d'annoncer que le Dr Paul M. O'Byrne, l'éminent professeur de l'Université McMaster (Hamilton, Ontario), présentera la Conférence honorifique de 2015 de la SCT lors du Congrès CHEST. Anciennement connu sous le nom de « Conférence commémorative Christie », ce prix reconnaît des contributions significatives au développement de la recherche, de l'éducation ou de l'excellence clinique dans le domaine de la pneumologie au Canada, ou au sein de la SCT. Félicitations au Dr O’Byrne!

Alors que le conseil d'administration et le Comité exécutif de la SCT s'occupent de la très nécessaire " troisième dimension » et de l'avenir de l'organisme, il est crucial de poursuivre des traditions d'importance comme celles-ci, qui célèbrent les contributions de nos membres à la profession et à l'amélioration de la santé pulmonaire au Canada. Au nom de la SCT, j'exprime ma sincère reconnaissance aux membres de nos comités permanents et assemblées cliniques, qui sont aux premières lignes de ces formidables efforts!

Le tout respectueusement soumis,

Diane Lougheed MD, M. Sc., FRCP

Présidente, Société canadienne de thoracologie 


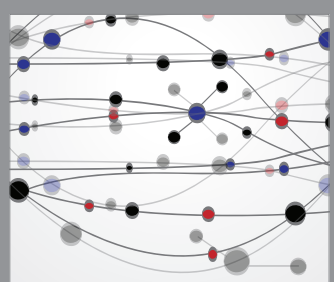

The Scientific World Journal
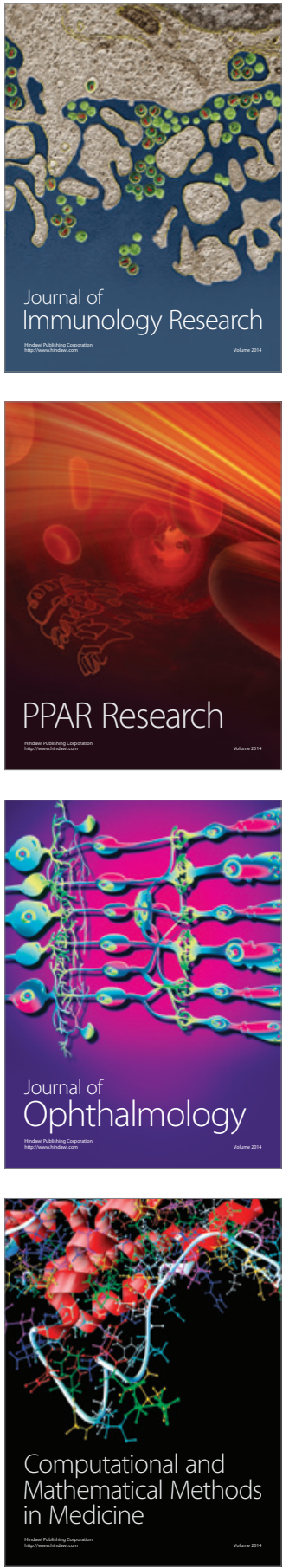

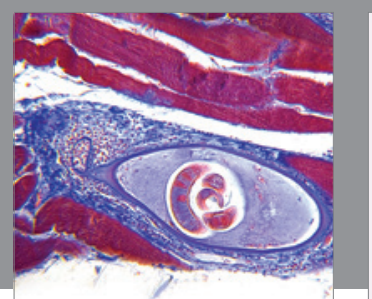

Gastroenterology Research and Practice

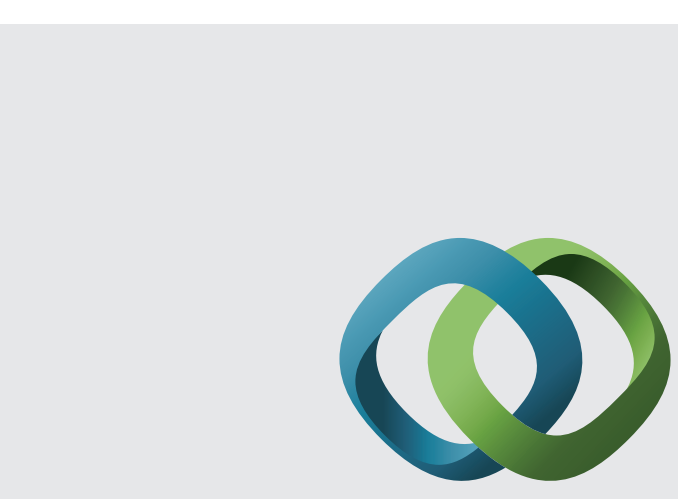

\section{Hindawi}

Submit your manuscripts at

http://www.hindawi.com
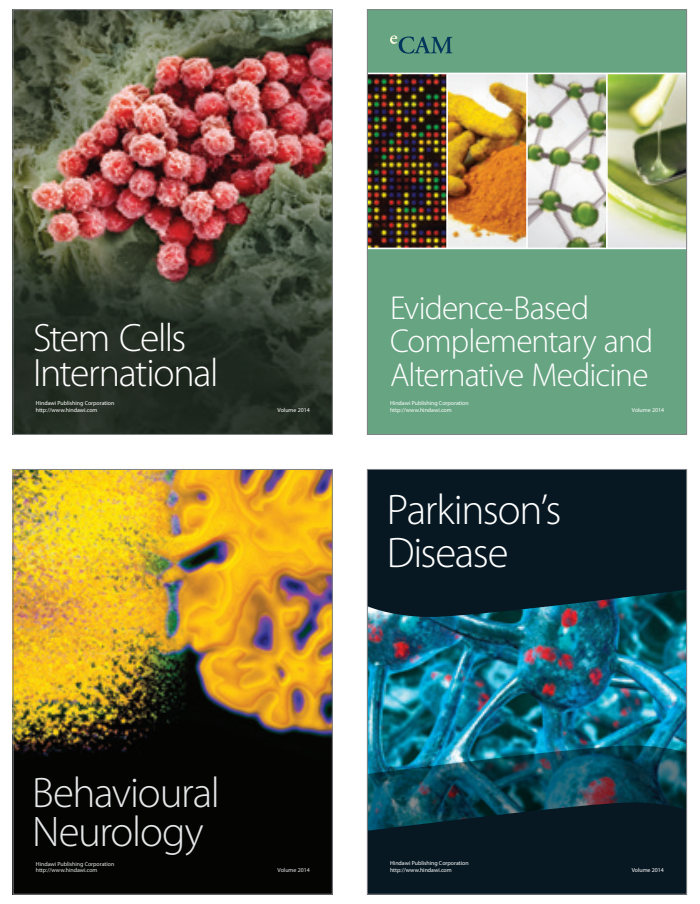
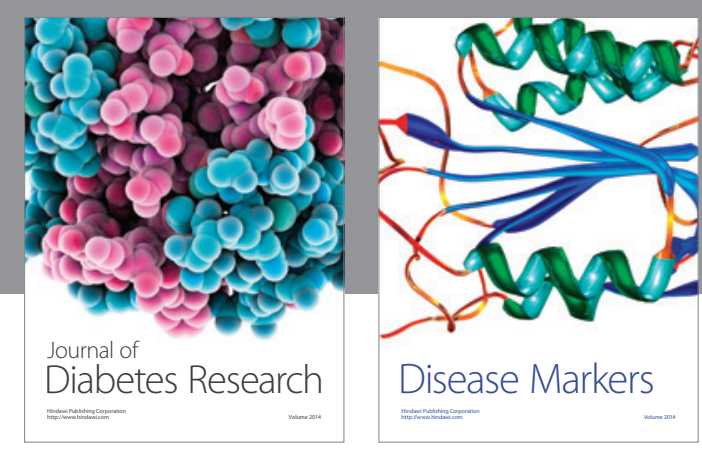

Disease Markers
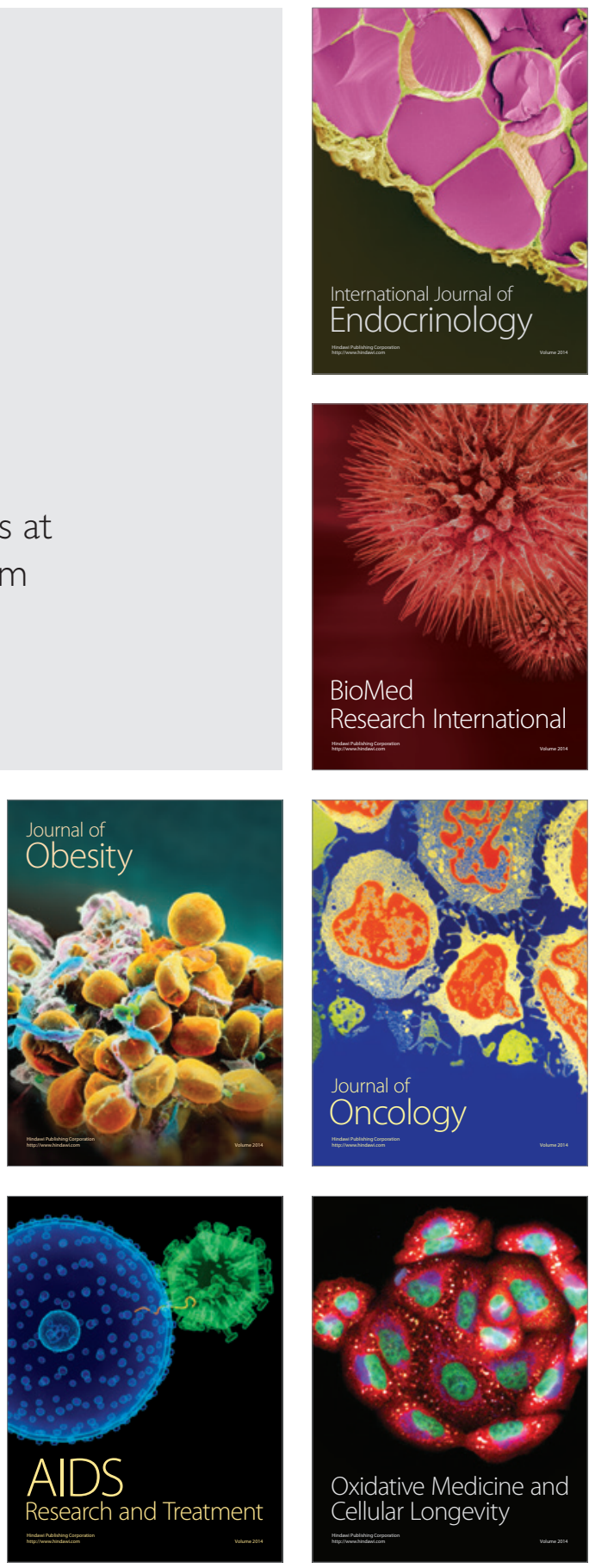